\title{
PERCEPCIÓN AMBIENTAL DE LOS VISITANTES A UN ZOOLÓGICO DE LIMA, PERÚ
}

\author{
José Iannacone $e^{1,2}$ \\ Lorena Alvariño ${ }^{2}$
}

\begin{abstract}
RESUMEN
Con fin de conocer la percepción ambiental de los visitantes a un zoológico de Lima, Perú, se realizó una encuesta de opinión basada en diez preguntas (cuatro respuestas cerradas y seis abiertas). Las encuestas fueron realizadas a 440 visitantes al zoológico del Patronato del Parque de las Leyendas (PATPAL) $\left(12^{\circ} 04^{\prime} 13^{\prime \prime} \mathrm{LS}, 77^{\circ} 04^{\prime 3} 9^{\prime \prime} \mathrm{LO}\right.$ y $\left.81 \mathrm{msnm}\right)$ el 10 de Julio del 2004. La proporción de sexos de los entrevistados fue aproximadamente 1:1. El grupo etario predominante fue mayor de 35 años $(31,1 \%)$. Solo el $20,45 \%$ de los entrevistados consideró al deterioro ambiental y a la contaminación como un problema de importancia que enfrenta la humanidad. Los entrevistados confundieron las causas del calentamiento global de la tierra $(68,44 \%$ ) y de la destrucción de la capa de ozono $(28,5 \%)$. Entre las funciones del zoológico se consideró la más importante la educativa, luego la de conservación y finalmente la de recreación. Manu (33,63\%), Paracas (14,09\%) y Pantanos de Villa (8,86\%) fueron las áreas Naturales protegidas por el Estado más mencionadas. La Vicuña fue la especie peruana más frecuentemente mencionada como en peligro de extinción. Solo 3,41 $\%$ señaló que en el Perú existen tres especies de aves marinas guaneras. En base a estos resultados obtenidos se propone algunos lineamientos de Educación Ambiental para los visitantes al zoológico.
\end{abstract}

Palabras claves: encuesta ambiental, percepción ambiental, Perú, visitantes, zoológico.

\section{SUMMARY}

In order to determine the environmental perception of visitors to a zoo in Lima, Peru, a survey of opinion based on ten questions (four open and six closed-ended) was performed. The surveys were conducted to 440 visitors to the Zoo Parque Las Leyendas (PATPAL) (12 ${ }^{\circ} 04^{\prime} 13^{\prime \prime} \mathrm{LS}, 77^{\circ} 04^{\prime} 39^{\prime \prime}$ and $\left.81 \mathrm{msnm}\right)$ on July 10, 2004. The sex ratio of respondents was approximately 1:1. The predominant age group was greater than 35 years $(31.1 \%)$. Only $20.45 \%$ of respondents considered environmental degradation and pollution as a major problem facing humanity. Respondents confused the causes of global warming of the earth $(68.44 \%)$ and the destruction of the ozone layer $(28.5 \%)$. The educational function of the zoo was considered the most important, also conservation and then finally, recreation. Manu (33.63\%), Paracas $(14.09 \%)$ and Villa Wetlands (8.86\%) were the natural areas protected by the state most often mentioned. The Peruvian Vicuña was the species most frequently mentioned as endangered. Only $3.41 \%$ said that in Peru there are three species of seabird guano. Based on these results some guidelines are proposed for environmental education for visitors to the zoo.

Key Words: environmental survey, environmental perception, Peru, visitors, zoo.

\section{INTRODUCCIÓN}

El ambiente es un sistema complejo en el que interactúan varios factores, recursos y condiciones relacionadas y entrelazadas entre sí. El ambiente se concibe como el desarrollo potencial basado en una articulación sinérgica de la productividad ecológica de los recursos naturales, en la productividad de los sistemas tecnológicos apropiados y en la productividad cultural que proviene de la movilización de los valores conservacionistas, de la creatividad social y de la diversidad cultural (POUEY etal., 2007; BRAGA, 2008).
La percepción ambiental se conceptualiza como la toma de conciencia del ambiente por el ser humano, es decir, es percibir el ambiente en el cual se localiza, aprendiendo a protegerlo y a cuidarlo de la mejor forma. La percepción ambiental establece una conexión entre el medio abiótico y una reflexión acerca de las relaciones de ese medio con la subjetividad, propia del instrumental psicológico de cada individuo (FAGGIONATO, 2005). El estudio de la percepción ambiental se direcciona a captar actitudes, conductas y visiones de los actores, en relación con los temas comprendidos en el marco del desarrollo sustentable. 
Comprende la visión del desarrollo, en su dimensión social, económica y ambiental (POUEY et al., 2007). La percepción ambiental de los individuos está constituida por toda una simbología fruto de su actividad cognitiva. Una parte importante de las respuestas perceptivas al ambiente se expresan por medio de juicios que entrañan evaluaciones cargadas de afecto, positivas o negativas a favor o en contra de determinados aspectos del ambiente. Las percepciones ambientales de distintos actores sociales adquieren formas particulares de comprender y apreciar el ambiente natural de acuerdo a un grupo social (BERTONI \& LÓPEZ, 2010).

Los parques zoológicos modernos presentan cuatro importantes metas: conservación, investigación, educación y recreación, estas dos últimas se encuentran directamente relacionadas con el visitante al zoológico (HARRIS, 1995; ANDERSON et al., 2003). Los zoológicos son secuencias de espacios que permiten a los visitantes ver, escuchar y oler constantemente a los animales que están comiendo, jugando, saltando como si estuvieran en su ambiente natural y observar su relación con otros animales e interactuar con el ambiente (TURLEY, 2001; HAM, 2007; YILMAZ et al., 2010). Los zoológicos representan un tipo de espacio abierto urbano que es reconocido por los visitantes para conectarse con la naturaleza, socializar, relajarse, aprender de los animales, ver animales exóticos, entretenerse y educar a los hijos, fotografiar la naturaleza y disfrutar del aire libre (TOMAS et al., 2002; TOFIELD et al., 2003; CLARK et al., 2005). Los zoológicos son cruciales en transmitir amor por la vida y la naturaleza, y cumplen con un esfuerzo global para educar a las personas, y por lo tanto pueden tener un impacto crucial en los visitantes (SIDERELIS \& GUSTKE, 2000; FALK et al., 2007; YILMAZ et al., 2010).

Las percepciones, actitudes y el comportamiento del visitante son herramientas importantes para evaluar las metas que tienen los zoológicos (ANDERSON et al., 2003). Los estudios de percepciones ambientales empleando encuestas en ambientes abiertos como las áreas naturales (GROFF et al., 2005; KALISCH \& KLAPHAKE, 2007; COSTA-NETO et al., 2010) y los zoológicos (MORGAN \& HODGKINSON, 1999; MONCADA et al., 2002; MYERS et al., 2003) son importantes para la elaboración de programas de educación ambiental (EA) pasivos y activos (D'AMATO \& KRASNY, 2011).

Por ende, el objetivo general del presente trabajo es determinar la percepción sobre la problemática ambiental en la población visitante a un zoológico de la Ciudad de Lima, Perú.

\section{MATERIAL Y MÉTODOS}

\section{Área de estudio:}

Fue realizado en el Zoológico del Patronato del Parque de las Leyendas (PATPAL) (12 $04^{\prime} 13^{\prime \prime}$ LS, $77^{\circ} 0439^{\prime \prime}$ LO y $81 \mathrm{msnm})$. El día de la encuesta se realizó en el zoológico, la celebración del Día del Maestro organizado por el Magisterio Peruano.

\section{Muestra:}

El tamaño muestral fue determinado a partir de los datos de afluencia promedio diaria proporcionada por la administración del PATPAL. El tipo de muestreo fue probabilístico debido a la intencionalidad en la selección de los entrevistados y a que la participación en la investigación era voluntaria por el visitante (IANNACONE \& ALVARIÑO, 2008; GONZÁLES et al., 2009). El diseño de investigación fue descriptiva, no experimental, de corte transversal y empleó el enfoque cuantitativo (FRICK \& ESPEJEL, 2006; MACEDO etal., 2010).

\section{Instrumento:}

La encuesta de percepción ambiental se llevó a cabo el 10 de julio del 2004 desde las 9:00 h hasta las 17:00 h (ocho horas de trabajo extramural). La encuesta fue realizada por 22 voluntarios previamente capacitados, que eran estudiantes de Post Grado de la Maestría de Gestión Ambiental de la Universidad Nacional Federico Villarreal (EUPG-UNV). A cada una de las 440 personas encuestadas, se le entrevistó entre 8 a 10 min durante su permanencia en el zoológico. El cuestionario estructurado constó de diez preguntas (cuatro cerradas y seis abiertas). Las preguntas incluyeron tres de tipología: edad, sexo y nivel de instrucción; tres sobre problemas globales: problemas que enfrenta la humanidad, calentamiento global de la tierra y destrucción de la capa de ozono (IANNACONE \& ALVARIÑO, 2006); cuatro sobre biodiversidad y conservación: funciones del zoológico (YILMAZ et al., 2010), áreas naturales protegidas, especies en peligro de extinción y aves guaneras. La encuesta fue validada por 22 expertos en el área ambiental y previamente se aplicó un muestreo piloto con 10 encuestas, permitiendo de este modo afinar el instrumento en el que finalmente se recabó la información (GONZÁLES et al., 2009). Para la formulación de las preguntas de la encuesta se consideró que tuvieran las siguientes características: que fueran claras, breves, precisas y sencillas, pertinentes, sin perjuicios, lógicas e inequívocas y que eviten ambigüedades y confusión.

La entrevista para la toma de los datos fue cara a cara (IANNACONE \& ALVARIÑO, 2008) y se desarrolló empleando los siguientes criterios: 1) el entrevistado 
debe encontrar la comodidad posible durante la encuesta, 2) fomento de confianza, 3) no se obligó a las personas a la entrevista, 4) las respuestas se obtuvieron natural y fielmente, 5) se evitó discusiones con el entrevistado, y 6) ante la negativa del encuestado a ser entrevistado se le agradecía y se optaba por otro candidato.

\section{Análisis de Datos:}

Los resultados son presentados en Tablas en base a las frecuencias o porcentajes de las respuestas a las preguntas planteadas en el cuestionario. Para determinar si la proporción sexual de los encuestados siguió la relación 1:1 se empleó el estadístico Chicuadrado $\left(\mathrm{X}^{2}\right)$ con corrección de Yates.

\section{RESULTADOS}

La Tabla 1 nos muestra que los grupos etarios de 35 a más años formaron el grupo mayoritario, luego el grupo entre 10 a 20 años conformaron el 38,9\% de los visitantes encuestados. La proporción sexual siguió la relación 1:1 $\left(\mathrm{X}^{2}=0,04, \mathrm{P}=0,84\right)$. Según el nivel de estudios alcanzado presentaron superior, secundaria, primaria y ninguna el $55 \%, 35 \%, 9,77 \%$ y $0,23 \%$, respectivamente.

Tabla 1. Distribución porcentual según sexo y grupo etario de 440 encuestados visitantes al Patronato Parque de Las Leyendas (PATPAL), Lima, Perú. N = Número de entrevistados.

\begin{tabular}{cccccccc}
\hline Edades & $\mathbf{1 0 - 1 4}$ & $\mathbf{1 5 - 2 0}$ & $\mathbf{2 1 -}$ & $\mathbf{2 6 -}$ & $\mathbf{3 1 -}$ & $\mathbf{3 5}$ a & \\
\hline \% en cada & Total \\
\hline $\mathrm{N}$ & 19,6 & 19,3 & 7,5 & 9,1 & 13,4 & 31,1 & 100 \\
Femenino (\%) & 87 & 85 & 33 & 40 & 59 & 136 & 440 \\
$\mathrm{~N}$ & 44,2 & 54,1 & 60,6 & 57,5 & 54,2 & 47,4 & 50,9 \\
Masculino (\%) & 38 & 46 & 20 & 23 & 32 & 65 & 224 \\
$\mathrm{~N}$ & 55,8 & 45,9 & 39,4 & 42,5 & 45,8 & 52,6 & 49,1 \\
& 49 & 39 & 13 & 17 & 27 & 71 & 216 \\
\hline
\end{tabular}

Con relación a los mayores problemas que enfrenta la humanidad, se observó que el problema de violencia se encuentra como primera opción, seguido del deterioro ambiental, pobreza y políticas de gobierno (Tabla 2).

Tabla 2. Respuesta de los 440 encuestados visitantes al Patronato Parque de Las Leyendas (PATPAL), Lima, Perú a la pregunta abierta: Cuales son los problemas a los que enfrenta la humanidad?

\begin{tabular}{lcc}
\hline Respuestas & $\mathbf{N}$ & $\mathbf{\%}$ \\
\hline Violencia & 137 & 21,88 \\
Deterioro ambiental y & & \\
contaminación & 128 & 20,45 \\
Pobreza & 100 & 15,97 \\
Políticas de gobierno & 95 & 15,18 \\
Falta de valores y cultura & 64 & 10,22 \\
Desempleo & 45 & 7,19 \\
Salud & 38 & 6,07 \\
Sobrepoblación & 14 & 2,24 \\
No responde & 5 & 0,80 \\
\hline Total & 626 & 100 \\
\hline
\end{tabular}

El número de respuestas es mayor a 440 debido a que varios de los encuestados respondieron más de una respuesta. 256, 130, 45 y 9 respondieron una, dos, tres y cuatro respuestas, respectivamente (Tabla 2 ).

La Tabla 3 nos muestra que casi el 50\% considera que las cuatro opciones de respuestas son las causas del calentamiento global. El 13,19\% ha considerado que el uso de insecticidas y de aerosoles como respuestas válidas como causas del calentamiento global.

Tabla 3. Respuesta de los 440 encuestados visitantes al Patronato Parque de Las Leyendas (PATPAL), Lima, Perú a la pregunta cerrada: Causas del calentamiento global de la tierra?

\begin{tabular}{lcc}
\hline Alternativas & N & $\mathbf{\%}$ \\
\hline Uso de insecticidas & 21 & 4,69 \\
Uso de aerosoles & 38 & 8,50 \\
Aumento de $\mathrm{CO}_{2}$ por el parque & 94 & 21,03 \\
automotor & & \\
Deforestación & 47 & 10,51 \\
Todas las anteriores & 223 & 49,88 \\
Ninguna de las anteriores & 24 & 5,37 \\
\hline Total & 447 & 100 \\
\hline
\end{tabular}

El número de respuestas es mayor a 440 debido a que varios de los encuestados respondieron más de una respuesta. 30, 384, 16, 9 y 1 respondieron cero, una, dos, tres y cuatro respuestas, respectivamente (Tabla 3 ).

Las tres principales consecuencias en orden decreciente de la destrucción de la capa de ozono fueron: 1) enfermedad en el ser humano, cáncer, ceguera y daños a la piel; 2) penetración de los rayos solares, y 2) calentamiento de la tierra (Tabla 4).

Tabla 4. Respuesta de los 440 encuestados visitantes al Patronato Parque de Las Leyendas (PATPAL), Lima, Perú a la pregunta abierta: Cuales son las consecuencias de la destrucción de la capa de ozono?

\begin{tabular}{lcc}
\hline Respuesta & $\mathbf{N}$ & $\mathbf{\%}$ \\
\hline Penetración de los rayos solares & 78 & 15,5 \\
Enfermedad en el ser humano, cáncer, ceguera, daños & & \\
a la piel & 191 & 37,9 \\
Contaminación ambiental & 29 & 5,7 \\
Calentamiento de la tierra & 75 & 14,8 \\
Efecto invernadero & 40 & 7,9 \\
Exterminio de la humanidad, guerras & 11 & 2,1 \\
Cambios climatológicos: desastres naturales & 19 & 3,7 \\
Destrucción de la vida en el planeta: muerte de & & \\
plantas, muerte de seres humanos & 22 & 4,3 \\
No sabe / no opina & 39 & 7,7 \\
\hline Total & 504 & 100 \\
\hline
\end{tabular}

El número de respuestas es mayor a 440 debido a que varios de los encuestados respondieron más de una respuesta. 5, 301, 109, 21, 1 y 3 respondieron cero, una, dos, tres, cuatro o cinco respuestas, respectivamente (Tabla 4). 
La Tabla 5 nos muestra cuales son las funciones del zoológico, considerándose la más importante la de educar: enseñar de los animales, conocer regiones y conocer la biodiversidad. Luego en segundo y tercer lugar se ubicaron proteger a los animales y plantas, fomentar crianza, desarrollo artificial del animal, y alimentación, y finalmente la recreación, respectivamente.

Tabla 5. Respuesta de los 440 encuestados visitantes al Patronato Parque de Las Leyendas (PATPAL), Lima, Perú a la pregunta abierta: Cual es función del zoológico?

\begin{tabular}{lcc}
\hline Respuesta & N & $\mathbf{\%}$ \\
\hline $\begin{array}{l}\text { Educativo: enseñar a los animales, conocer regiones y } \\
\text { conocer la biodiversidad }\end{array}$ & 207 & 47,04 \\
Proteger a los animales y plantas, fomentar crianza, & & \\
desarrollo artificial del animal, alimentación & 162 & 36,81 \\
Recreación & 59 & 13,41 \\
Ser hábitat & 1 & 0,23 \\
Oxigenar ciudades & 2 & 0,45 \\
No sabe / No opina & 9 & 2,04 \\
\hline Total & 440 & 100 \\
\hline
\end{tabular}

La Tabla 6 nos indica que los tres lugares que presentaron los mayores porcentajes como áreas naturales protegidas por el estado peruano $(56,58 \%) \mathrm{se}$ encuentran en esas categorías. El 11,8\% citó erróneamente al Parque de las Leyendas y a otros parques distritales como áreas naturales protegidas por el estado. Inclusive algunos citaron a las Huacas, líneas de Nazca y Rioja como áreas Naturales protegidas por el Estado.

Tabla 6. Respuesta de los 440 encuestados visitantes al Patronato Parque de Las Leyendas (PATPAL), Lima, Perú a la pregunta abierta: Cuales son los lugares considerados como áreas naturales protegidas por el estado peruano?

\begin{tabular}{lcr}
\hline Respuestas & N & \% \\
\hline Manu & 148 & 33,63 \\
Paracas & 62 & 14,09 \\
Pantanos de Villa & 39 & 8,86 \\
Parque de Las Leyendas & 26 & 5,90 \\
Otros Parques Distritales & 26 & 5,90 \\
Pacaya- Samiria & 22 & 5,00 \\
Otros (menos de siete citaciones) & 82 & 18,63 \\
no conoce & 35 & 7,96 \\
\hline Total & 440 & 100 \\
\hline
\end{tabular}

Con relación a la especie de animal Peruano en peligro de extinción, las respuestas estuvieron muy dispersas, citaron a la vicuña, al gallito de las rocas, al oso de Anteojos, a la alpaca, a la llama, y al cóndor (Tabla 7). En la lista fueron citadas con términos generales las aves (1 citación), aves de selva (1 citación), osos (1 citación), tortugas (1 citación) y auquénidos (2 citaciones). Fueron citadas especies introducidas naturalizadas como la trucha (2 citaciones). De igual forma se señalaron como animales peruanos en peligro de extinción a especies de otros continentes como el elefante (1 citación), león (1 citación), osos polares (3 citaciones), foca (4 citaciones) y oso panda (10 citaciones).

Tabla 7. Respuesta de los 440 encuestados visitantes al Patronato Parque de Las Leyendas (PATPAL), Lima, Perú a la pregunta abierta: Señale una especie Animal Peruana en Peligro de extinción?

\begin{tabular}{lcr}
\hline Alternativas & N & \% \\
\hline Vicuña & 82 & 18,63 \\
Gallito de las Rocas & 37 & 8,41 \\
Oso de Anteojos & 25 & 5,68 \\
Alpaca & 23 & 5,22 \\
Llama & 19 & 4,32 \\
Cóndor & 16 & 3,63 \\
Lobo de mar & 11 & 2,50 \\
Oso Panda & 10 & 2,27 \\
Cocodrilo de Tumbes & 6 & 1,36 \\
Delfín & 6 & 1,36 \\
Jaguar & 6 & 1,36 \\
Mono Tigre & 6 & 1,36 \\
Tigrillo & 5 & 1,13 \\
Foca & 4 & 0,90 \\
Guanaco & 4 & 0,90 \\
Mono Aullador & 4 & 0,90 \\
Puma & 4 & 0,90 \\
Taruca & 4 & 0,90 \\
Otras especies (menos de & 107 & 24,31 \\
4 citaciones) & & \\
No sabe, no recuerda & 61 & 13,86 \\
\hline
\end{tabular}

El mayor porcentaje consideró que solo existe una especie de ave guanera, seguido de cero y dos especies respectivamente (Tabla 8). De igual forma cuando se les preguntó que cite una especie de aves guanera, se observo la siguiente secuencia: Pelicano $(n=77$; $17,5 \%)>$ guanay $(n=49 ; 11,13 \%)>$ piquero $(n=27$; $6,13 \%)>$ gaviota $(n=16 ; 3,63 \%)>$ pingüino $(n=9$; $2,05 \%)$ y no sabe fue la mayoría $(n=268)(60,90 \%)$.

Tabla 8. Respuesta de los 440 encuestados visitantes al Patronato Parque de Las Leyendas (PATPAL), Lima, Perú a la pregunta cerrada: Cuál es el número de especies de aves consideradas como guaneras?

\begin{tabular}{ccc}
\hline Respuesta & Frecuencia & \% \\
\hline 0 & 99 & 22,27 \\
1 & 283 & 64,32 \\
2 & 42 & 9,55 \\
3 & 15 & 3,41 \\
4 & 2 & 0,45 \\
más de 4 & 0 & 0 \\
\hline
\end{tabular}

\section{DISCUSIÓN}

\section{Problemas Globales}

Con relación a los mayores problemas que enfrenta la humanidad, se observó que el problema de violencia se encuentra como primera opción, seguido del deterioro ambiental, pobreza y políticas de gobierno. IANNACONE \& ALVARIÑO (2006) encontraron en un grupo de participantes en un taller de comunicación 
en el zoológico del PATPAL durante el 2005 que entre los problemas ambientales globales más importantes a la contaminación y a la pobreza, citados en el presente estudio como la segunda y tercera opción.

Los resultados en nuestro estudio muestran que muchos visitantes al zoológico de Lima confundieron la problemática del calentamiento global y destrucción de la capa de ozono. Así el 50\% considera que las cuatro opciones de respuestas fueron las causas del calentamiento global. El 13,19\% consideró que el uso de insecticidas y de aerosoles como respuestas válidas como causas del calentamiento global. También entre las tres principales consecuencias en orden decreciente de la destrucción de la capa de ozono citaron al calentamiento de la tierra. Estos resultados fueron similares a lo señalados por OLTRA et al. (2009) quienes encontraron en un grupo de participantes con relación al conocimiento del cambio climático (SOLÍS, 2006), confundieron el calentamiento global y la destrucción de la capa de ozono (SADPI, 2007).

\section{Biodiversidad y Conservación}

Entre las funciones del zoológico se consideró la más importante la educativa, luego la de conservación y finalmente recreación. YILMAZ et al. (2010) encontró de igual forma en un estudio de percepción de visitantes que las principales razones para visitar un zoológico eran conocer de los animales (educativo) y para el entretenimiento y relajación. KAZAROV (2008) argumenta que el rol más importante de los zoológicos es crear una ética de conservación en los visitantes.

Los tres lugares que presentaron los mayores porcentajes de citación como áreas naturales protegidas por el estado peruano para el 2004 (56,58\%): Parque Nacional del Manu (D.S. N. ${ }^{\circ} 045-$ 2002-AG), Reserva Nacional de Paracas (D.S. N. ${ }^{\circ} 045-$ 2002-AG) y Zona Reservada de los Pantanos de Villa (RMC 00144-89-AGdGTT) considerado actualmente refugio de vida silvestre, se encuentran en esas categorías. El 11,8\% citó erróneamente al Parque de las Leyendas y a otros parques distritales como áreas naturales protegidas por el estado. Inclusive algunos citaron a las Huacas, líneas de Nazca y Rioja como áreas Naturales protegidas por el Estado, siendo estas últimas no vinculadas a las 56 áreas naturales del INRENA-2004 (LÓPEZ, 2004).

Con relación a la especie de animal Peruana en peligro de extinción, las respuestas estuvieron muy dispersas, citaron a la vicuña (Vicugna vicugna), al gallito de las rocas (Rupicola peruviana), al oso de Anteojos (Tremartos ornatus), a la alpaca (especie doméstica), a la llama (especie doméstica), y al cóndor (Vultur gryphus). Para el periodo que se realizó la encuesta estaba vigente para el Perú la resolución D.S. No $013-$ 99-G del 19 de mayo de 1999, donde se incluyeron como en vías de extinción al Cocodrilo de Tumbes, Guanaco, Taruca y Oso de anteojos; en vulnerable a la vicuña, gallito de las rocas, condor andino, lobo de mar, tigrillo, jaguar u otorongo, mono titi o leoncillo y mono aullador. La "tortuga" que incluye varias especies se encuentran citadas en las especies indeterminadas. En la lista fueron citadas con términos generales las aves, aves de selva, osos, tortugas y auquénidos. Fueron citadas especies introducidas exóticas, pero naturalizadas como la trucha (Oncorhynchus mykiss). De igual forma se señalaron como animales peruanos en peligro de extinción a especies de otros continentes megacarismáticos como el elefante, león, osos polares, foca y oso panda.

El mayor porcentaje consideró que solo existe una especie de ave guanera, seguido de cero y dos especies respectivamente. También cuando se les preguntó que cite una especie de aves guanera, la secuencia fue: pelicano, guanay y piquero (FIGUEROA \& STUCCHI, 2008). Sin embargo, también citaron a aves no guaneras como la gaviota y al pingüino, y los que no sabían fueron la mayoría $(60,90 \%)$.

El grado de percepción de los visitantes sobre la biodiversidad y ambiente son elementos decisivos en el éxito o fracaso en los esfuerzos de conservación que dependen del nivel de escolaridad, estrato etario y social (COSTA-NETO et al., 2010; SCHRAM, 2011; WHITE et al., 2011).

Es muy importante implementar programas de EA (FRICK \& ESPEJEL, 2006) que empleen procedimientos de interpretación de la naturaleza para sensibilizar al visitante sobre las problemáticas ambientales y de biodiversidad existentes en el Patronato del Parque de las Leyendas (ZAMORA, 2007; SCHRAM, 2011), elaborando guías de visita con un lenguaje apropiado al perfil del visitante tomando en cuenta sus actitudes a la biodiversidad (MARTINO, 2008; COSTA et al., 2010). ZARAGOZA et al. (2009) señalan que una función de la EA es la de facilitar la utilización de la información socioambiental en la resolución de problemas y establecer la relación entre la ciencia y los actores sociales involucrados en el manejo de ecosistemas, sus recursos y los servicios que proveen. También la EA es considerada una actividad mediadora para la construcción de sociedades sostenibles.

\section{LITERATURA CITADA}

ANDERSON, S., KELLING, PRESSLEYKEOUGH, R., BLOOMSMITH, M.A., MAPLE, T.L. 2003. Enhancing the zoo visitor's experience by public animal training and oral interpretation at an otter exhibit. Environment and Behaviour. 35 : 826-841. 
BERTONI, M. \& LÓPEZ, M.J. 2010. Percepciones sociales ambientales. Estudios y Perspectivas en Turismo. 19: 835-849.

BRAGA, F.S. 2008. A geografia humanística e suas relações com o ecoturismo. Revista Universitaria de Geografía. 17: 105-124.

COSTA, C.C., OLIVEIRA, I.S.S. \& GOMES, L.J. 2010. Percepción ambiental como estrategia para el ecoturismo en Unidades de Conservación. Estudios y perspectivas en Turismo. 19: 11211135.

COSTA-NETO, A.R., TELLO, J.R., COSTA, L.A., VIANA, A.L., ALVES, J.L. \& BUHRING, R. 2010. Gestão dos espaços naturais de Manaus: uma interpretação da sensibilização e participação publica na conservação de áreas protegidas. Acta Amazonica. 40: 667-674.

CLARK, R.S., LIVINGSTON, M. \& SMITH, S.E. 2005. Visitor behavior in zoo exhibits with underwater viewing. Visitor Studies Today. 8: 1-10.

D'AMATO, L.G. \& KRASNY, M.E. 2011. Outdoor adventure education: applying transformative learning theory to understanding instrumental learning and personal growth in Environmental Education. The Journal of Environmental Education. 42: 237-254.

FAGGIONATO, S. 2005. Percepção Ambiental. Disponible en: http://educar.sc.usp.br/ biologia/textos /m_a_txt4.html, 2005. Visitado el: 25 de octubre de 2011 .

FALK, J.H., REINHARD, E.M., VERNON, C.L., BRONNENKANT, K. \& HEIMLICH, J.E. 2007. Why zoos \& Aquarium matter: Assessing the impact of a visit. Association of Zoos \& Aquariums. Silver Spring, MD.

FIGUEROA, J. \& STUCCHI, M. 2008. Las aves de las islas Lobos de Afuera (Perú) en la primavera del 2004. Ornitología Neotropical. 19: 377-390.

FRICK, B.A. \& ESPEJEL, B.O. 2006. Semiótica ambiental, y gestión comunitaria. Horizontes Antropológicos. 12: 257-269.

GONZÁL EZ, A., MONCADA， J.A. \& ARANGUREN, J. 2009. Los visitantes del Parque zoológico y botánico Bararida, Estado de Lara: Demanda real e implicaciones educativas ambientales. Investigación y Postgrado. 24: 213238.

GROFF, A., LOCKHART, D. \& OGDEN, J. \& DIERKING, L.D. 2005. An exploratory investigation of the effect of working in an environmentally themed facility on the conservation-related knowledge, attitudes and behavior of staff. Environmental Education Research. 11:371-387.

HAM, S. 2007. Environmental interpretation: a practical guide for people with big ideas and small budgets. North American Press. Colorado. USA. $52 \mathrm{p}$.
HARRIS, L. 1995. Recreation in a zoo environment: applying animal behavior research techniques to understand how visitors allocate time. Legacy. 6: 14-18.

IANNACONE， J. \& ALVARIÑO, L. 2006. Incorporación de la Educación Ambiental en las áreas curriculares en Perú: Experiencias de docentes en un Taller de Comunicación con énfasis en Toxicología Ambiental. Revista de Ciencias (Lima). 3: 23-44.

IANNACONE，J. \& ALVARIÑO, L. 2008. Percepción de la problemática socioeconómica y ambiental entre los pobladores residentes del Lago Junín, Perú. Revista de Ciencias (Lima). 5:21-37.

KALISCH， D. \& KLAPHAKE，A. 2007. Visitors'satisfaction and perception of crowding in a German National Park: a case study on the island of Halling Hooge. Forest Snow and Landscape Research. 81: 109-122.

KAZAROV, E. 2008. The role of zoos in creating a conservation ethic in visitors. Washington University in St. Louis. Environmental Studies: Social Sciences Australia, Sydney. 47 p.

LÓPEZ, L.A. 2004. Iberoamérica y sus sistemas de áreas naturales protegidas el caso de Perú. Observatorio Medioambiental. 7: 97-115.

MARTINO, D. 2008. Gender and urban perceptions of nature and protected areas in Bañados del Este Biosphere Reserve. Environmental Management. 41: 654-662.

MACEDO, R.F. \& DANTAS, A.V.S. 2010. Percepción de los turistas sobre el uso de los recursos sócio-ambientales de la playa do Meio en Natal (RN) - Brasil. Estudios y Perspectivas en Turismo. 19: 636-672.

MONCADA, J.A., ARANGUREN, J. \& DIAZ, E. 2002. Implicaciones prácticas de las preferencias de los visitantes del Parque Zoológico Caricuao, Caracas. Investigación y Desarrollo. 17: 135-158.

MORGAN, J.M. \& HODGKINSON, M. 1999. The motivation and social orientation of visitors attending a contemporary zoological park. Environment and Behaviour. 31:227-239.

MYERS, O.L. Jr., SAUNDERS, C.D.\& GARRETT, E. 2003. What do children think animals need? Aesthetic and psycho-social conceptions. Environmental Education Research .9: 305-325.

OLTRA, C., SOLÁ, R., SALA, R., PRADES, A. \& GAMERO, N. 2009. Cambio climático: percepciones y discurso público. Prismasocial. 2: 1-23.

POUEY, N., FERRERO, A.B., OMELIANIUK, S., TAVARES, R. \& ZANUCOLI, M. 2007. La percepción ambiental como instrumento de gestión para la protección y desarrollo sostenible del sistema acuífero Guaraní: aplicación piloto en Federación, entre Ríos. 16 p. XVII Simposio Brasileiro de Recursos Hídricos, Brasil. 
SCHRAM, H. 2011. Looking at people looking at animals. An international bibliography on visitor experience studies and exhibit evaluation in zoos and aquariums. EAZA Education Committee. Version O.3 dd. 10 February 2011.272 p.

SIDERELIS, C. \& GUSTKE, L. 2000. Influence of on-site choices on recreation demand. Leisure Sciences. 22: 123-132.

SADPI (Socialdata Australia for Department for planning and Infrastructure). 2007. Climate change in-depth Perth. Survey of environment perceptions, knowledge and behaviours of 108 households in the cities of Mandurah and Canning. Perth, Western Australia. 52 p.

SOLIS, A.I. 2006. Gases de efecto invernadero y cambio climático. Revista de Ciencias (Lima). 3: 75-82.

TOFIELD, S., COLL, R.K., VYLE, B. \& BOLSTAD, R. 2003. Zoos as a source of free choice learning. Research in Science \& Technological Education. 21: 67-99.

TOMAS, S.R., SCOTT, D. \& CROMPTON, J.L. 2002. An investigation of the relationships between quality service performance, benefits sought, satisfaction and future intention to visit among visitors to a zoo. Managing Leisure. 7: 239-250.

TURLEY, S.K. 2001. Children and the demand for recreational experience: the case of zoos. Leisure Studies. 20: 1-18.
WHite, T.H., CAMACHO, A.J., BLOOM, T., DIÉGUEZ, P.L. \& SELLARES, R. 2011. Human perceptions regarding endangered species conservation: a case study of Saona Island, Dominic Republic. Revista Latinoamericana de Conservación. 2: 18-29.

YILMAZ, S., MUMCU, S. \& ÖZBILEN, A. 2010. Effects of spatial difference on visitor perceptions at zoo exhibits. Scientific Research and Essay. 5: 2327-2340.

ZAMORA, B.N. 2007. El INBioparque como un espacio educativo-recreativo para la conservación de la biodiversidad. Análisis de la percepción de usuarios. pp. 169-186. En: Nuevas Tendencias en investigaciones en Educación Ambiental. Naturaleza y Parques Nacionales. Serie educación ambiental. Secretaría General para el Territorio y la Biodiversidad. Organismo Autónomo Parques Nacionales. Ministerio del Ambiente. Diploma de Estudios Avanzados. Universidad Autónoma de Madrid y Universidad Nacional de Costa Rica.

ZARAGOZA, R.M., BOADA, M., BARRERABASSOLS, N. \& McCALL, M. 2009. Percepciones socioambientales infantiles $y$ adolescentes. Propuesta de educación ambiental, La Huacana (Michoacán, México). Utopía y Praxis Latinoamericana. 14:39-51. 